\title{
Proyecto interdisciplinario en la enseñanza de la ingeniería para el fortalecimiento de las ciencias básicas
}

\section{Interdisciplinary project in engineering teaching for the strengthening of basic sciences}

PAZ-GONZÁLEZ, Juan Antoniơ, URIARTE-RAMÍREZ, Irma, ROSAS-BURGOS, Virginia Karina, MORALES-CONTRERAS, Óscar Adrián

Universidad Autónoma de Baja California, Facultad de Ciencias de la Ingeniería, México.

ID $1^{\text {er }}$ Autor: Juan Antonio, Paz-González / ORC ID: 0000-0002-3807-5011, Researcher ID Thomson: AAN-2299-2020 CVU CONACYT ID: 298818

ID $1^{\text {er }}$ Coautor: Irma, Uriarte-Ramírez / ORC ID: 0000-0002-9442-8731, Researcher ID Thomson: ANN-1113-2020 CVU CONACYT ID: 671766

ID $2^{\text {do }}$ Coautor: Virginia Karina, Rosas-Burgos / ORC ID: 0000-0001-7232-0204, Researcher ID Thomson: AAN-18852020, CVU CONACYT ID: 670301

ID $3^{\text {er }}$ Coautor: Óscar Adrián, Morales-Contreras / ORC ID: 0000-0003-0118-8132, arXiv Author ID: aomc07, CVU CONACYT ID: 49211

DOI: $10.35429 /$ JHS.2020.11.4.11.21

Recibido Enero 15, 2020; Aceptado Junio 30, 2020

\section{Resumen}

En este artículo se propone una metodología para desarrollar un proyecto de aprendizaje interdisciplinario, en donde los estudiantes de Tronco Común de Ciencias de la Ingeniería (TCI) seleccionen un proceso productivo de acuerdo con el perfil profesional de la ingeniería de su interés del cual identificarán las competencias y conocimientos principales que éste demande y los relacione con los conocimientos teóricoprácticos desarrollados en los cursos de ciencias básicas. El objetivo es construir nuevas estructuras de conocimiento por medio de la integración de las diferentes disciplinas que forman el primer semestre de ingeniería para dar solución a un problema en común, en este caso, el proceso productivo. De acuerdo a las tendencias educativas y la sociedad de la información, cada vez más las instituciones de educación superior utilizan el aprendizaje de forma interdisciplinaria, ya que es notorio en el entorno académico un cambio en la manera de enseñar y aprender. Otro beneficio de esta propuesta es contrarrestar la apatía y desánimo que se percibe en los estudiantes ya que de manera inmediata no visualizan los propósitos de las unidades de aprendizaje debido a que las perciben como independientes.

Proyecto interdisciplinario, ciencias básicas, educación en ingeniería

\begin{abstract}
This article proposes a methodology to develop an interdisciplinary learning project, where the student of first Semester of General Education of Engineering Sciences selects a productive process according to the professional profile of the engineering of his interest from which he will identify the main competences and knowledge that he demands and relate them to the theoretical and practical knowledge developed in the courses of basic sciences. The objective is to build new knowledge structures through the integration of the different disciplines that make up the first semester of engineering to provide solutions to a common problem, in this case, the productive process. In accordance with educational trends and the information society, higher education institutions are increasingly using learning in an interdisciplinary way, since a change in the way of teaching and learning is evident in the academic environment. Another benefit of this proposal is to fight the apathy and discouragement that is perceived in students since they do not immediately visualize the purposes of the learning units because they perceive them as independent.
\end{abstract}

Interdisciplinary project, basic sciences, engineering education

Citación: PAZ-GONZÁLEZ, Juan Antonio, URIARTE-RAMÍREZ, Irma, ROSAS-BURGOS, Virginia Karina, MORALES-CONTRERAS, Óscar Adrián. Proyecto interdisciplinario en la enseñanza de la ingeniería para el fortalecimiento de las ciencias básicas. Revista de Educación Superior. 2020. 4-11:11-21.

\footnotetext{
$\uparrow$ Investigador contribuyendo como primer autor.
} 


\section{Introducción}

El contexto del proyecto es el de una universidad pública, cuyo modelo educativo, contempla tres atributos esenciales: la flexibilidad curricular, la formación integral y el sistema de créditos. Estos atributos permean cinco componentes interrelacionados que conforman el modelo educativo: el aprendizaje centrado en el alumno, el enfoque por competencias, las modalidades de aprendizaje, la extensión y vinculación y la movilidad. En conjunto, estos componentes están presentes a lo largo del proceso formativo (Cuamea, Álvarez, Reyes, Cervantes, \& Martínez, Modelo Educativo de la Universidad Autónoma de Baja California, 2006).

En este modelo, los estudiantes ingresan a un proceso de homologación de conocimientos llamado Tronco Común perteneciente a la etapa formativa básica. Esta etapa comprende un proceso general de carácter interdisciplinario, con una orientación formativa al incorporar elementos contextualizadores, metodológicos e instrumentales esenciales para la formación del futuro profesionista ingenieril (Cuamea, Álvarez, Reyes, Cervantes, \& Martínez, Modelo Educativo de la Universidad Autónoma de Baja California, 2006).

Es común que, durante una jornada académica, y debido a la flexibilidad curricular, los estudiantes cambien de grupo, profesor y asignatura; esto deriva en que los alumnos intenten trabajar y aprender los temas de las cartas descriptivas que los profesores imparten, sin detenerse a pensar en cuáles de estos temas deben de ser profundizados o no, o peor aún, se enfrentan a la exigencia de tareas y proyectos de acuerdo con el modelo antiguo de enseñanza.

Por su parte en Humes, (2013), se explica "no hay ocupación en donde los trabajadores deban de cambiar de trabajo cada hora, moverse a otro lugar de trabajo, y trabajar bajo la dirección de un supervisor diferente en cada cambio. Sin embargo, es lo que precisamente les pedimos a nuestros jóvenes, esperando al mismo tiempo, de proveerles un programa educativo coherente".
Para dar solución a la problemática antes mencionada, se propone una metodología a los estudiantes del primer semestre de ingeniería, que les permita el desarrollo de un proyecto de aprendizaje interdisciplinario que integre los contenidos de cinco materias de tres disciplinas de las ciencias: matemáticas, química y las humanidades.

El proyecto interdisciplinario como medio para desarrollar competencias básicas en el estudiante de ingeniería, es una forma de hacer partícipe a los estudiantes de su propio aprendizaje, al incorporar a los alumnos a este tipo de desafíos; así ellos se comprometen y se identifican como especialistas en una de las disciplinas integradas en el proyecto y participan activamente en el equipo y en el desarrollo de este.

El aprendizaje interdisciplinario y multicontextual es una de las actividades que promueve y ejercita habilidades primordiales. Se pueden incluir el aprendizaje colaborativo, el aprendizaje basado en problemas e investigación basada en la acción. El énfasis está dado por las habilidades transferibles que pueden ser aplicadas a través de diferentes áreas del conocimiento, también es sabido que algunas habilidades pueden ser específicas de un tema (Humes, 2013).

El aprendizaje interdisciplinario se encuentra conectado y directamente relacionado a experiencias de la vida real, ya que promueve el pensamiento crítico y el desarrollo de habilidades para el aprendizaje del trabajo cotidiano (Humes, 2013).

Los estudios interdisciplinarios se definen como el proceso necesario para responder una pregunta, resolver un problema o debatir un tema que es muy amplio o complejo para ser trabajado por una sola disciplina conlleva a perspectivas disciplinarias e integra sus puntos de vista para producir un entendimiento más comprensivo o un avance más cognitivo (Repko, 2008), como lo sería el afrontar los retos y cambios de la sociedad, ambiente y cultura tanto en el presente como en el futuro (Reyes Suárez, 2020). 
Una de las ventajas de un proyecto de forma interdisciplinaria es que en ocasiones es necesario buscar información extra clase; lo que promueve contribuciones significativas al dominio textual personal, capacidad de razonamiento matemático, salud y estado mental. En el dominio textual hay oportunidades para usar diferentes fuentes literarias, utilizar y entender tecnicismos, entender tablas, mapas, calendarios, instrucciones habladas y comprensión de manuales de uso. En lo que respecta al razonamiento matemático, hay oportunidades de medir ángulos y calcular distancias, por ejemplo.

En salud y estado mental hay oportunidades para llegar a ser físicamente activo en diferentes maneras $\mathrm{y}$, por ende, mejorar el estado emocional y de salud mental (Scottish Government, 2010).

Otra ventaja de este proyecto y metodología interdisciplinaria es que el alumno involucra el saber de las ciencias básicas con las de las aplicadas y así percibe el surgimiento de algunas de las ramas de la ingeniería con enfoque multi e interdisciplinario como ejemplos: Energías Renovables, Bioingeniería, Ingeniería Aeroespacial o Mecatrónica que son carreras de la oferta educativa. El corazón de la interdisciplinariedad es la comunicación, es decir; las conversaciones, conexiones y combinaciones que traen nuevos desafíos a cada tipo de científico e ingeniero (Sciences, 2004). Este trabajo está dividido en cinco secciones: en II se presenta una descripción del rol del docente en esta propuesta, en III se describe la misma, en IV la discusión de los resultados y finalmente en $\mathrm{V}$ las conclusiones.

\section{El rol del docente en el proyecto interdisciplinario}

Un profesionista al convertirse en docente debe poseer competencias personales y profesionales específicas con el propósito de desarrollar y cumplir las demandas que la sociedad exige, por esa razón los futuros docentes deben de prepararse de manera multidimensional, interdisciplinar y transdisciplinar.
En este sentido, el convertirse en profesor de una universidad implica la participación de cada uno en el desarrollo profesional de los estudiantes y de ellos mismos. En este tipo de esquema se espera que el facilitador, como es considerado al docente en el modelo educativo, promueva la interacción dialógica, horizontal, la relación entre pares, en el contexto de que cada persona sea tomada en cuenta como un ser cognoscente, capaz de construir y reconstruir su propio reconocimiento. Es decir, debe intervenir activamente para su propio aprendizaje, esto le permitirá transformar sus conocimientos y experiencias para desarrollar sus competencias como futuro profesionista (Chacón-Corzo, Chacón, \& Alcedo, 2012).

Los docentes actuales deben contribuir a enfatizar la importancia e integración entre las diversas disciplinas, de manera que cada profesor pueda edificar interrelaciones, romper esas barreras que imponen los limites disciplinares y entender la realidad educativa en el carácter multicontextual y complejo. Esto conlleva, que los proyectos interdisciplinarios deben nacer del contexto y responder a las necesidades y problemas inherentes a dicha realidad, lo que origina la integración de saberes y el análisis para la resolución de problemas y transformación social (ChacónCorzo, Chacón, \& Alcedo, 2012).

Otros autores han señalado cambios significativos en la práctica docente después de la incorporación de proyectos interdisciplinarios en el aula, los cuales se mantienen en un periodo de intervención. Ellos afirman que se puede observar una reconstrucción de un concepto de aprender y enseñar más cercano a un paradigma constructivista y se manifiesta una consistencia entre el discurso y la práctica de los profesores. También se observó que los profesores fueron progresivamente incorporando nuevas prácticas en su quehacer a la vez que le dan un nuevo significado a las definiciones que otorgan a sus clases (Denegri-Coria, 2005). 
Se han publicado diferentes ventajas sobre los proyectos interdisciplinarios como sistemas estratégicos educativos en los estudiantes de educación superior con el propósito de que dichos alumnos puedan adquirir competencias y habilidades con una visión más amplia a la que actualmente se tiene si se aprendiera o se especializara en una sola disciplina (Davidovitch, 2013) (Budd \& Dumas, 2014), dichos trabajos han enfatizado la diferencia entre una educación multidisciplinaria y una interdisciplinaria. En el caso de la educación multidisciplinaria esta se basa en la unión de campos especializados en una manera no integrativa, es decir, reteniendo las metodologías y teorías de cada disciplina, mientras que, en el caso de una educación basada en la interdisciplinariedad, ésta involucra el cruce de las características de las disciplinas tradicionales a un nuevo campo, con un objetivo en común, necesitando de competencias académicas provenientes de dos o más áreas de conocimiento.

El objetivo básicamente de un proyecto interdisciplinario entonces es el crear un avance cognitivo que no es razonablemente anclado a una sola disciplina (Davidovitch, 2013). Tal es el caso de la carrera de Mecatrónica, una ingeniería que utiliza conocimientos de control, electrónica e informática para realizar innovaciones en los procesos industriales. $\mathrm{La}$ Universidad de Ariel, en Israel, reportó el uso de la educación multidisciplinaria y discutió cómo ésta afecta a la sociedad, dicha publicación menciona que adoptando este tipo de sistemas en la educación superior se pueden visualizar cambios en el conocimiento, investigación, y reflejos educativos que impactan a la población, dichos cambios incluyen una alteración conceptual que refleja el cambio en la manera de cómo piensan los individuos (Davidovitch, 2013).

Una de las ventajas fundamentales de un proyecto ya sea multidisciplinario $o$ interdisciplinario es que cuando se exponen los avances de un proyecto que se está realizando de manera interdisciplinaria o multidisciplinaria, los expositores se ven obligados a explicar fundamentos y estrategias, presentando ideas pertenecientes a una disciplina en particular a un público no conocedor, por lo que tiene que digerir la información y hacerla explícita al público (Budd \& Dumas, 2014).

\section{El proyecto interdisciplinario}

Con el propósito de favorecer el trabajo interdisciplinario, así como alcanzar el aprendizaje significativo se solicitó a los estudiantes del Tronco Común de Ingeniería (TCI), de la Facultad de Ciencias de la Ingeniería y Tecnología, Universidad Autónoma de Baja California (FCITECUABC), unidad Valle de las Palmas; como parte de la evaluación semestral, la presentación de un proyecto final con el cumplimiento de la metodología y criterios de evaluación correspondientes, a fin de asegurar el logro de las competencias establecidas en los siguientes cursos: Introducción a la Ingeniería, Comunicación Oral y Escrita, Química, Álgebra Lineal y Cálculo Diferencial. El proyecto a continuación se describe.

\section{Contexto}

El proyecto fue llevado a cabo por alumnos del primer semestre de TCI, los guías de este proyecto, fueron los responsables de academia de Ciencias Básicas, así como los profesores que imparten las materias. Los cursos participantes en el proyecto pertenecen a tres academias: química (química general), matemáticas (álgebra lineal, cálculo diferencial) y humanidades (comunicación oral y escrita e introducción a la ingeniería). Éstas ubicadas en el plan de estudios en el primer semestre del TCI. Por conveniencia, ya que el desarrollo de un proyecto es parte del contenido temático, se eligió al curso de introducción a la ingeniería y por ende los docentes, coordinadores del proyecto. Los alumnos se integraron en equipos de trabajo, idealmente de cinco estudiantes y del mismo perfil profesional de interés (carrera en ingeniería que desea cursar). Para el caso de los estudiantes que estaban cursando por segunda ocasión alguna(s) las unidades de aprendizaje involucradas en el proyecto interdisciplinario, se integraron a un equipo especial, en donde se le dio seguimiento a la metodología, con énfasis en documentar y exponer la aplicación de los conocimientos de la(s) asignatura(s) pendiente(s) de acreditar. 


\section{Método}

Una vez definido el equipo de trabajo, éste se desarrolló en cada una de las etapas de la metodología descrita en la Figura 1.

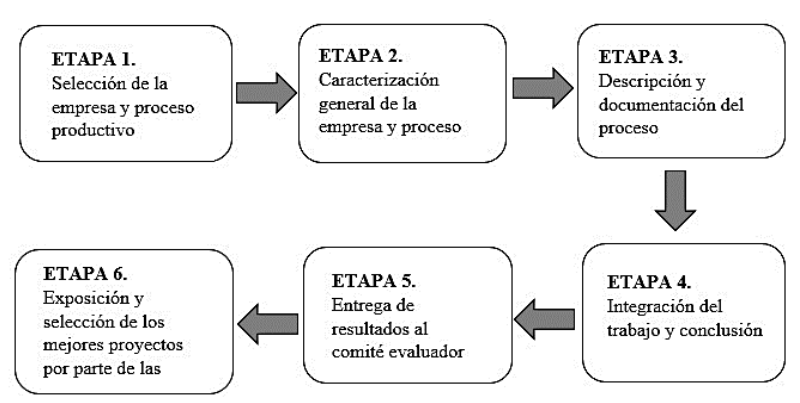

Figura 1 Descripción de las etapas del proyecto interdisciplinario

Fuente: elaboración propia

\section{Etapa 1}

De común acuerdo, los integrantes seleccionaron una empresa en la cual reconocieron las aplicaciones profesionales de la ingeniería de interés del equipo. A través de investigación documental, el equipo trabajó en la redacción del objetivo general y los objetivos específicos del proyecto. En esta etapa también se seleccionó el proceso productivo (de fabricación, diseño, línea de producción, ciclo de vida del producto o aplicación) que le empresa en cuestión maneja. A los estudiantes se les proporcionaron temas considerando el desarrollo de un producto relacionado a una ingeniería ofertada por la institución (Tabla 1).

\begin{tabular}{|l|l|}
\hline \multicolumn{1}{|c|}{ Ingeniería } & \multicolumn{1}{c|}{ Proyecto } \\
\hline Bioingeniería & Stent/ Ortesis de cadera \\
\hline Mecánica & Engranes/ Materiales compuestos \\
\hline Aeroespacial & Alas/ Turbinas \\
\hline $\begin{array}{l}\text { Energías } \\
\text { Renovables }\end{array}$ & Panel solar/ Aerogeneradores \\
\hline Mecatrónica & $\begin{array}{l}\text { Embotelladoras/ Brazos } \\
\text { robóticos }\end{array}$ \\
\hline Electrónica & $\begin{array}{l}\text { Sistemas de comunicación/ } \\
\text { Automatización de procesos }\end{array}$ \\
\hline Civil & Canales/ Puentes \\
\hline Industrial & Metrología/ Control de calidad \\
\hline Eléctrica & Motores/ Estaciones eléctricas \\
\hline
\end{tabular}

Tabla 1 Descripción de los temas de cada equipo considerando el desarrollo de un producto relacionado a una ingeniería de la universidad

Fuente: elaboración propia

\section{Etapa 2}

En esta etapa, el equipo trabajó en la recopilación de información que describe la empresa seleccionada: antecedentes, misión, visión, y giro económico principal. Así como la descripción general de las áreas o departamentos en donde se desarrolla el proceso productivo seleccionado.

\section{Etapa 3}

El equipo aplicó el proceso de diseño en ingeniería, las herramientas para el análisis matemático, químico y gráfico de información, así como datos utilizados en ingeniería para realizar la descripción del proceso de interés.

La información recopilada debió cumplir con los criterios de validación y confiabilidad de las fuentes de información.

Este proceso consta de las siguientes etapas: observación y recopilación de información, registro, análisis y resultados. Este proceso se estudia en el contenido temático de la materia Introducción a la Ingeniería. Entre las herramientas solicitadas se encuentran: el histograma, diagrama de Pareto, diagrama de flujo, diagrama causa-efecto y el análisis estadístico.

\section{Etapa 4}

El equipo de trabajo presentó una conclusión sobre el proyecto destacando los hallazgos más relevantes, aportando ideas o recomendaciones, así como la expresión de lo aprendido en el trabajo interdisciplinario. Lo esperado por el cuerpo colegiado evaluador, es que los estudiantes identifiquen los conocimientos de las ciencias básicas en los procesos de ingeniería investigados. A continuación, se describen los objetivos específicos por materia.

\section{Introducción a la Ingeniería (II)}

Identificar algunas de las actividades más relevantes del ejercicio profesional asociadas a la ingeniería de su interés, a través de la descripción de un proceso de fabricación desarrollado en un sistema productivo real, utilizando las herramientas matemáticas, tecnológicas y de análisis de información estudiadas en el curso; con una actitud crítica, objetiva y responsable. 


\section{- Comunicación Oral y Escrita (COyE)}

Aplicar las técnicas de comunicación, utilizando los conocimientos teóricos y prácticos de la expresión oral, escrita y corporal, para mejorar la capacidad de escuchar, expresar tanto ideas como experiencias, con una actitud de tolerancia y respeto hacia las personas.

\section{- Química General (QG)}

Describir las propiedades fisicoquímicas fundamentales de la materia, para predecir el comportamiento y área de aplicación de los materiales y sustancias químicas en los procesos industriales y/ o productos, utilizando el material y equipo de medición básico de química y las herramientas teóricas de la Estequiometría; participando proactivamente en equipos de trabajo, con objetividad, tolerancia y respeto; atendiendo las reglas de seguridad e higiene y cuidando el medio ambiente.

\section{- Álgebra Lineal (AL)}

Emplear el sistema de los números complejos, y el álgebra matricial, mediante la aplicación de sus distintas representaciones y propiedades de operación, para resolver e interpretar problemas cotidianos y de ingeniería, con actitud reflexiva, disposición para el trabajo colaborativo, responsabilidad y tolerancia.

\section{- Cálculo Diferencial (CD)}

Aplicar los conceptos y procedimientos del cálculo en la diferenciación de funciones, mediante el uso de límites y teoremas de derivación, apoyados en tecnologías de información, para resolver problemas cotidianos, de ciencia e ingeniería, con disposición para el trabajo colaborativo, respeto y honestidad.

\section{Etapa 5}

El equipo de trabajo entrega un informe de proyecto final en manuscrito y en formato de cartel científico. Se entrega el informe del proyecto a cada uno de los miembros del comité evaluador. En el caso de las asignaturas de Introducción a la Ingeniería y Comunicación Oral y Escrita, se utilizó la herramienta SafeAssignment de Blackboard para evaluar la originalidad en los documentos.
Los equipos de estudiantes entregaron dos evidencias para la evaluación del proyecto, estas incluyen un informe del proyecto, el cual es un manuscrito con un formato definido que contiene: portada, índice de contenido, resumen, una introducción y objetivo general, la descripción de la empresa (antecedentes y descripción de área o departamento donde se desarrolla el proceso de fabricación), la descripción del proceso de estudio, utilizando la metodología Osborn: Observación y recopilación, Registro, Análisis y Resultados, para la identificación de aplicaciones relacionadas con los cursos de Comunicación Oral y Escrita, Química, Álgebra Lineal y Cálculo Diferencial. Aplicación de herramientas gráficas de análisis (estudiadas en Introducción a la ingeniería), para la descripción del proceso de fabricación seleccionado, conclusión y referencias.

\section{Etapa 6}

En esta etapa final, los grupos de estudiantes son evaluados de manera oral por el comité. Durante todas las etapas del proyecto, los estudiantes son asesorados por el comité evaluador, el cual está formado por cuatro profesores de las diferentes disciplinas del conocimiento. Las actividades se realizaron mediante un cronograma que fue entregado a los estudiantes al inicio de la actividad.

\section{Herramientas para la evaluación del proyecto interdisciplinario}

En la Figura 2 se observa el formato de evaluación, este formato fue entregado a cada uno de los miembros del comité evaluador. Es pertinente mencionar que la calificación del proyecto estuvo distribuida de la siguiente manera: el documento escrito con un valor del $70 \%$ y la exposición oral con un $30 \%$, el cual equivale al $25 \%$ en la evaluación final de cada una de las materias participantes. Esta calificación se decidió de manera colegiada. Como se mencionó, participaron en este proyecto tres academias pertenecientes al TCI. En reuniones colegiadas, se llegó al acuerdo de la calificación del proyecto. Estas ponderaciones y calificaciones se dieron a conocer a los estudiantes al inicio del semestre verbalmente y por escrito por medio del encuadre de clase. 


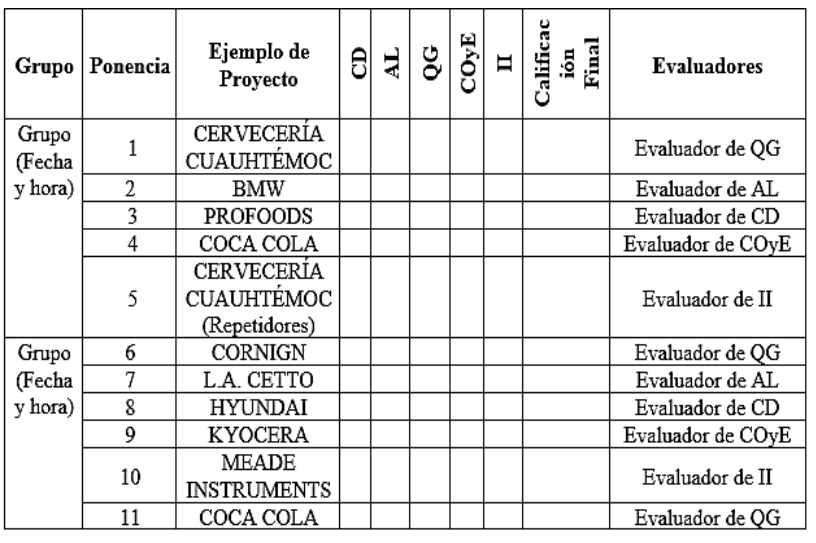

Figura 2 Tabla de calificaciones para cada exposición y grupo. Cálculo diferencial (CD). Álgebra Lineal (AL). Química General (QG). Comunicación Oral y Escrita (COyE). Introducción a la Ingeniería (II)

Fuente: Elaboración propia

Este formato sirvió para procesar la valoración del proyecto interdisciplinario por cada integrante del comité. Para la calificación final se obtuvo un promedio global el cual corresponde al porcentaje establecido en el encuadre para cada una de las Academias (Química, Humanidades y Matemáticas)

Cualquier duda de la metodología, los estudiantes se acercaron con el profesor titular de cada una de las involucradas en el proyecto.

\section{Resultados y discusiones}

Para obtener resultados con base al aprendizaje y puntos de vista de los participantes del proyecto (estudiantes y profesores) se realizaron 50 encuestas a estudiantes de TCI, donde el $74 \%$ son hombres y el $26 \%$ son mujeres, cuyas edades fluctúan entre los 19 años hasta los 27 años, $24 \%$ del segundo semestre y $76 \%$ de primer semestre. Entre las carreras a las cuales dichos estudiantes pretenden ingresar después de haber acabado el TCI se encuentran las siguientes preferencias: $15 \%$ ingeniería mecatrónica, $27 \%$ Bioingeniería, $12 \%$ Ingeniería industrial, $12 \%$ de ingeniería mecánica, $18.94 \%$ ingeniería aeroespacial, $15 \%$ ingeniería civil y solo un $0.06 \%$ ingeniería eléctrica. En el caso de los profesores se realizaron 10 encuestas a los docentes que participaron en la evaluación del proyecto interdisciplinario cuyas asignaturas, participaron en el proyecto. Entre los alumnos encuestados que se encuentran en TCI, el $24 \%$ está repitiendo alguna de las cinco asignaturas evaluadas en la actividad (Gráfico 1).

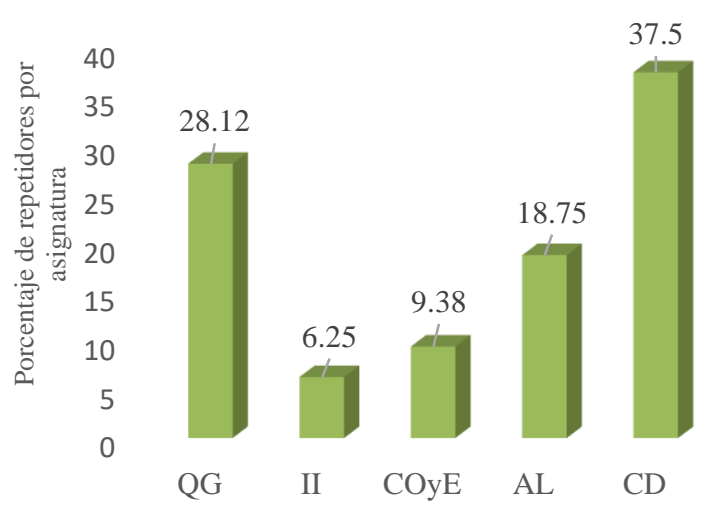

Gráfico 1 Porcentaje de repetidores por asignatura de primer semestre de Tronco Común de Ingeniería

Fuente: Elaboración propia

Se puede visualizar que las materias de Matemáticas y Química son las que causan mayores índices de reprobación de los estudiantes. En el Gráfico 2 se muestran las asignaturas que resultaron con mayor dificultad para ser integradas en el proyecto interdisciplinario.

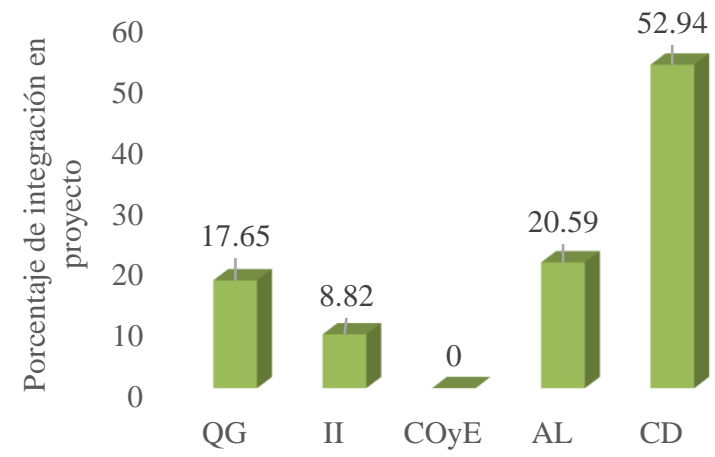

Gráfico 2 Porcentaje de dificultad de integración en el proyecto interdisciplinario

Fuente: Elaboración propia

Estas cifras manifiestan que las materias que incluyen cálculos y razonamiento matemáticos son las que implican mayor dificultad a los estudiantes para relacionar los conceptos teóricos con la aplicación real en los procesos de ingeniería. Tanto en matemáticas como en química, este es un factor que provoca apatía y posiblemente deserción de los alumnos de la carrera. De acuerdo con (Sánchez-Amaya, Navarro Salcedo, \& García-Valencia, 2009) una de las causas principales de la reprobación y deserción de los estudiantes son los factores personales, los cuales se expresan en un $51.1 \%$ de desmotivación, un $21.3 \%$ de insatisfacción académica, un $8.5 \%$ de problemas de salud, 6.4 $\%$ de desadaptación, un $4.3 \%$ de problemas emocionales y otro tipo de causas representadas en un $8.5 \%$. 
Los investigadores de Sánchez-Amaya, Navarro Salcedo, \& García-Valencia, (2009) afirman que dentro de estos factores puede definirse otra categoría: malestar personal $(78.8 \%)$, en la cual confluyen, la desmotivación, la insatisfacción académica y la desadaptación que reflejan una indisposición y una desesperanza del estudiante que no se siente a gusto con lo que estudia, con la institución e incluso con los compañeros y docentes, lo cual ocasiona unos niveles bajos de comprensión, desinterés y apatía.

Algunos otros trabajos en la literatura especializada han reportado varios motivos que dan origen a la apatía; gestión del tiempo y autorregulación, independencia, autoconcepto, confianza, vergüenza, toma de decisiones estratégicas y motivación (Uchida, 2010).

Otro de los factores que se puede destacar que provocan la apatía en los estudiantes, es la percepción que ellos tienen sobre la utilidad de sus asignaturas en algún semestre respectivo. Sin embargo, las encuestas realizadas a los estudiantes demuestran que ellos comprenden la utilidad de las matemáticas en sus ingenierías, un $73.53 \%$ de los encuestados opinan que las asignaturas de matemáticas (álgebra lineal y cálculo diferencial), son indispensables para su desarrollo como ingenieros (Gráfico 3).

A pesar de esto, este tipo de asignaturas son las que poseen los más altos índices de reprobación en las universidades que ofertan ingenierías.

Aunque para algunos de los estudiantes participantes fue complicado relacionar los conceptos de la química y las matemáticas en los procesos elegidos, este ejercicio del proyecto interdisciplinario, es una estrategia propuesta por las academias para combatir la apatía, la desmotivación e incluso el desinterés o indiferencia por las ciencias básicas, ya que los estudiantes consideran que están disociadas con respecto a las materias propias de la disciplina que se van a estudiar en la siguiente etapa de formación.
El proyecto interdisciplinario es un factor importante para que los alumnos puedan visualizar la aplicación real de las competencias aprendidas en las materias de TCI, de hecho, el $82.35 \%$ obtuvo un panorama mucho más amplio de lo que implica la ingeniería que van a estudiar y el $79.41 \%$ afirma que este tipo de ejercicios son importantes para el primer semestre ya que desarrolla en ellos competencias tales como la organización del tiempo, toma de decisiones, uso de herramientas digitales, comunicación interpersonal, trabajo en equipo, negociación, creatividad, etc. Estas competencias pertenecen a las llamadas competencias genéricas sistémicas que se busca desarrollar en esta etapa de formación. (Cuamea, Álvarez, Reyes, Cervantes, \& Martínez, Modelo Educativo de la Universidad Autónoma de Baja California, 2006).

Se ha reportado que la apatía entre los estudiantes universitarios está ligada directamente con el compromiso y responsabilidad hacia las actividades académicas solicitadas por los docentes (Schoen \& Fusarelli, 2008). En muchas universidades, los docentes culpan al estudiante de ser apático y no interesarle la escuela. Sin embargo, al entrevistar a estudiantes ellos culpan a la institución de no darles la importancia adecuada del mismo aprendizaje del estudiante (Thompson \& Allen, 2011). Es importante destacar que si no se combate la apatía de los estudiantes aun cuando estos sean competentes y cuentan con todos los recursos a su disposición, la institución educativa no puede hacer nada al respecto para llevar al estudiante hacia un aprendizaje óptimo (Baines \& Slutsky, 2009).

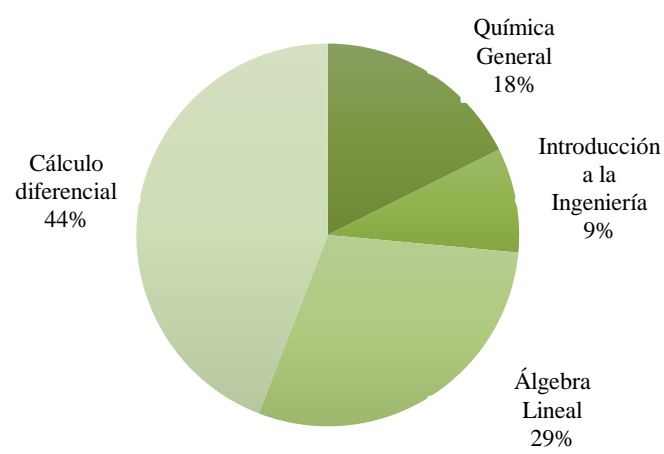

Gráfico 3 Asignaturas que los alumnos aseguran que tienen más importancia para sus ingenierías Fuente: Elaboración propia 
Otro de los factores a considerar es el desarrollo de la habilidad para el trabajo en equipo, en este tipo de actividad, el $26.47 \%$ menciona que sólo alguno de los compañeros realizó el trabajo y no todo el equipo, el $8.82 \%$ de los equipos sólo lo trabajo un integrante y el $64.71 \%$ afirma que trabajaron todos los integrantes del equipo. El 55.88 \% afirmó que no tuvo dificultades para trabajar en equipo.

En la evaluación del proyecto, en la sesión oral, participó un comité evaluador interdisciplinario. Esto con el fin de que ésta fuera una evaluación objetiva. El $17.65 \%$ de los alumnos se sintieron desafiados, el $14.75 \%$ de los alumnos se sintieron frustrados, el 11.76 $\%$ indiferentes, el $52.94 \%$ se consideró tranquilo y sólo el $2.94 \%$ se intimidó. Se afirmó el $67.65 \%$ que la evaluación a su proyecto fue más objetiva e imparcial al ser evaluado por profesores de diferentes asignaturas.

En la Tabla 2, se pueden apreciar los temas de cada asignatura que los estudiantes pudieron encontrar relación o lograron utilizar las competencias adquiridas en clase en el desarrollo del proyecto interdisciplinario.

\begin{tabular}{|l|l|}
\hline \multicolumn{1}{|c|}{ Asignaturas } & \multicolumn{1}{c|}{ Temas Involucrados } \\
\hline Álgebra Lineal & $\begin{array}{l}\text { Números polinomiales, matrices, } \\
\text { vectores, producto punto, producto } \\
\text { cruz, ecuaciones lineales, formula de } \\
\text { Euler y gráficas de proporcionalidad. }\end{array}$ \\
\hline $\begin{array}{l}\text { Cálculo } \\
\text { Diferencial }\end{array}$ & $\begin{array}{l}\text { Derivadas, límites, resolución de } \\
\text { desigualdades, gráficas de funciones } \\
\text { trigonométricas, desplazamiento } \\
\text { rectilíneo, movimientos básicos de } \\
\text { un punto, mínimos, álgebra general, } \\
\text { desigualdades y su solución, } \\
\text { funciones y sus gráficas, dominio y } \\
\text { rango de una función, limites, } \\
\text { derivadas, desplazamiento rectilíneo. }\end{array}$ \\
\hline $\begin{array}{l}\text { Comunicación } \\
\text { Oral y Escrita }\end{array}$ & $\begin{array}{l}\text { Redacción, objetividad, } \\
\text { comunicación, manera de exponer, y } \\
\text { contenido de los documentos, } \\
\text { comunicación en las empresas, tener } \\
\text { comunicación con otros individuos, } \\
\text { identificar las partes de un texto, } \\
\text { redacción, como hacer una buena } \\
\text { presentación, forma, comunicación } \\
\text { no verbal, lenguaje corporal, } \\
\text { comunicar y trasmitir información, } \\
\text { expresión oral y corporal, facilidad } \\
\text { de la palabra, ortografía y el tener un } \\
\text { buen habla, tildes y gramática. }\end{array}$ \\
& \multicolumn{1}{|c|}{} \\
\hline & \\
\hline
\end{tabular}

\begin{tabular}{|c|c|}
\hline $\begin{array}{l}\text { Introducción a } \\
\text { la Ingeniería }\end{array}$ & $\begin{array}{l}\text { Procesos, historia de la ingeniería, } \\
\text { diagrama de flujo, herramientas } \\
\text { tecnológicas, características de un } \\
\text { ingeniero, diagrama de Pareto, } \\
\text { antecedentes, identificación de } \\
\text { ingenierías, el objetivo de las } \\
\text { ingenieras, conversiones, conocer las } \\
\text { ingenieras, formación de un mapa o } \\
\text { diagrama de flujo de un proceso, } \\
\text { herramientas en la ingeniería, } \\
\text { matemáticas, graficas e ingeniería. }\end{array}$ \\
\hline $\begin{array}{l}\text { Química } \\
\text { General }\end{array}$ & $\begin{array}{l}\text { Enlaces, moléculas, moles, } \\
\text { reacciones químicas, reactivo } \\
\text { limitante, densidad, relación masa } \\
\text { volumen, composición, proceso de } \\
\text { combustión, rendimiento de } \\
\text { reacción, neutralización, fórmulas } \\
\text { químicas (balanceo de ecuaciones), } \\
\text { reacciones que se tienen en quema } \\
\text { de gasolina, compuestos, balanceo } \\
\text { de ecuaciones, combinación de } \\
\text { soluciones, masa molar, mezcla de } \\
\text { los elementos, fermentación, } \\
\text { concentraciones químicas, } \\
\text { conversiones, estequiometria. }\end{array}$ \\
\hline
\end{tabular}

Tabla 2 Temas de cada asignatura que se vieron involucradas en el proyecto interdisciplinario Fuente: Elaboración propia

En lo que se refiere a los docentes, todos afirman que la exposición de los alumnos con temas interdisciplinarios integrados en un mismo proyecto, fue más completo e ilustrativo que una exposición para cada materia. Asimismo, los decentes reportaron que el $50 \%$ de los estudiantes solicitaron asesoría debido al interés de los alumnos por resolver un problema real.

El $30 \%$ afirma que el $25 \%$ de los alumnos se acercaron a preguntar sobre los temas relacionados a su materia para integrarlo en el proyecto durante este semestre, otro $30 \%$ mencionó que el $75 \%$, otro $30 \%$ comentó que el $50 \%$ y solo uno contestó que nadie se le acerco a preguntar.

El $60 \%$ de los docentes afirmó que al exponer los temas los alumnos lograron integrar satisfactoriamente su materia en el proyecto interdisciplinario y el $90 \%$ visualizó líderes de grupos utilizando esta estrategia de aprendizaje. El $80 \%$ de los docentes encuestados afirmó que por lo menos uno de los proyectos presentados fue creativo y satisfactorio. El $60 \%$ de los docentes afirmaron no tener preferencia positiva o negativa al calificar a sus propios alumnos en comparación a los alumnos que no fueron sus estudiantes. El $90 \%$ recomendó continuar implementando esta actividad.

PAZ-GONZÁLEZ, Juan Antonio, URIARTE-RAMÍREZ, Irma, ROSAS-BURGOS, Virginia Karina, MORALES-CONTRERAS, Óscar Adrián. Proyecto interdisciplinario en la enseñanza de la ingeniería para el fortalecimiento de las ciencias básicas. Revista de Educación Superior. 2020 


\section{Conclusiones}

El proyecto interdisciplinario es una herramienta para desarrollar y alcanzar las competencias planteadas en la etapa básica de las diferentes carreras en ingeniería que se ofertan en la FCITEC-UABC, desarrollando en el estudiante la capacidad de relacionar un proceso productivo con las disciplinas que interactúan en él, a través de una metodología dada y generando en el estudiante conciencia de las responsabilidades sociales que adquiere un ingeniero en la toma de decisiones. Por medio de los proyectos interdisciplinarios, los estudiantes se acercaron al campo laboral del ingeniero y al panorama de las estructuras que se deben de llevar en la producción. Esto lo faculta para tener una visión clara y realista sobre el campo laboral regional, así como de la oferta y demanda de ingenieros en las diferentes empresas. Este ejercicio permitió al estudiante, concretar la elección de la ingeniería a la que se incorporará en la siguiente etapa de su formación académica.

Así también, el estudiante comprendió la importancia de las materias de las ciencias básicas, la aplicación real, la relación de éstas con los procesos reales en la ingeniería y cómo éstas trabajan de manera conjunta para describir proceso de manufactura de diferentes productos que se ofrecen en el mercado. Otro punto importante fue que, a través del proyecto, se observó interrelación entre las unidades de aprendizaje, donde cada una de ellas se aplica en un mismo proceso, pero con un enfoque diferente que dan como resultado un producto o una operación, todas teniendo como objetivo mejorar el proceso y aumentar la eficiencia desde distintos puntos de vista, dentro de los cuales se incluyen diferentes áreas de las ingenierías, por lo que todas las ingenierías tienen la misma importancia en la elaboración de productos.

Aun así, luego de reflexionar sobre el resultado del proyecto, en contraste con el apartado teórico; se considera importante avanzar de la interdisciplina hacia la transdisciplina, dados los retos actuales que tienen en los distintos entornos (industrial, social, ambiental y económico, entre otros); mediante el diálogo con las ciencias humanas; así como incorporar desde la etapa básica, la enseñanza del desarrollo sostenible como un eje transversal.

\section{Referencias}

Baines, L. A., \& Slutsky, R. (2009). Developing the Sixth Sense: Play. Educational Horizons, 97-101.

Budd, J., \& Dumas, C. (2014). Epistemic Multiplicity in Schools: Expanding Knowledge through Interdisciplinarity. The Canadian Journal of Information and Library Science, 271-286.

Chacón-Corzo, M., Chacón, C., \& Alcedo, Y. (2012). Los proyectos de aprendizaje interdisciplinarios en la formación docente. Revista Mexicana de Investigación Educativa, 877-902.

Cuamea, F., Álvarez, J., Reyes, J. C., Cervantes, O., \& Martínez, L. (2006). Modelo Educativo de la Universidad Autónoma de Baja California. Mexicali: UABC.

Cuamea, F., Álvarez, J., Reyes, J. C., Cervantes, O., \& Martínez, L. (2006). Modelo Educativo de la Universidad Autónoma de Baja California. Mexicali: UABC.

Davidovitch, N. (2013). Paradigmatic changes in perceptions of disciplinary and multidisciplinary teaching in Israel higher education system: fad or challenge? Israel Affairs, 704-712.

Denegri-Coria, M. (2005). Proyectos de aula Interdisciplinarios y Reprofesionalización de profesores: un modelo de capacitación. Estudios Pedagógicos, 82-93.

Humes, W. (2013). Curriculum for Excellence and Interdisciplinary Learning. Scottish Educational Review., 82-93.

Repko, A. (2008). Interdisciplinary Research: Process and Theory. London: Sage.

Reyes Suárez, M. (2020). Secuencia didáctica para el fortalecimiento del pensamiento.

Rodríguez Núñez, L. H., \& Londoño Londoño, F. J. (2011). Estudio sobre deserción estudiantil en los programas de Educación de la Católica del Norte Fundación Universitaria. Revista Virtual Universidad Católica del Norte, 1-28. 
Sánchez-Amaya, G., Navarro Salcedo, W., \& García-Valencia, A. (2009). Factores de Deserción Estudiantil en la Universidad Surcolombiana. Paideia Surcolombiana, 97103.

Schoen, L., \& Fusarelli, L. (2008). Innovation, NCLB, and the fear factor: The challenge of leading 21st-century schools in an era of accountability. Educational Policy, 181-201.

Sciences, N. A. (2004). Facilitating Interdisciplinary Research. Washington, DC: National Academies Press.

Thompson, G., \& Allen, T. (2011). Four effects of the high-stakes testing movement on African American K-12 students. The Journal of Negro Education, 218-227.

Uchida, C. (2010). Apathetic and withdrawing students in Japanese universities with regard to Hikikomori and student apathy. Journal of Mesical and Dental Sciences, 95-108. 